\title{
Evidence of carbamate resistance in urban populations of Anopheles gambiae s.s. mosquitoes resistant to DDT and deltamethrin insecticides in Lagos, South-Western Nigeria
}

Adedayo O Oduola ${ }^{1,2^{*}}$, Emmanuel T Idowu², Muyiwa K Oyebola², Adedapo O Adeogun ${ }^{2}$, Judith B Olojede ${ }^{1}$, Olubunmi A Otubanjo ${ }^{2}$ and Taiwo S Awolola ${ }^{1}$

\begin{abstract}
Background: Resistance monitoring is essential in ensuring the success of insecticide based vector control programmes. This study was carried out to assess the susceptibility status of urban populations of Anopheles gambiae to carbamate insecticide being considered for vector control in mosquito populations previously reported to be resistant to DDT and permethrin.

Methods: Two - three day old adult female Anopheles mosquitoes reared from larval collections in 11 study sites from Local Government Areas of Lagos were exposed to test papers impregnated with DDT 4\%, deltamethrin $0.05 \%$ and propoxur $0.1 \%$ insecticides. Additional tests were carried out to determine the susceptibility status of the Anopheles gambiae population to bendiocarb insecticide. Members of the A. gambiae complex, the molecular forms, were identified by PCR assays. The involvement of metabolic enzymes in carbamate resistance was assessed using Piperonyl butoxide (PBO) synergist assays. The presence of kdr-w/e and ace-1R point mutations responsible for DDT-pyrethroid and carbamate resistance mechanisms was also investigated by PCR.

Results: Propoxur resistance was found in 10 out of the 11 study sites. Resistance to three classes of insecticides was observed in five urban localities. Mortality rates in mosquitoes exposed to deltamethrin and propoxur did not show any significant difference $(P>0.05)$ but was significantly higher $(P<0.05)$ in populations exposed to DDT. All mosquitoes tested were identified as A. gambiae s.s ( $M$ form). The $k d r-w$ point mutation at allelic frequencies between 45\%-77\% was identified as one of the resistant mechanisms responsible for DDT and pyrethroid resistance. Ace-1R point mutation was absent in the carbamate resistant population. However, the possible involvement of metabolic resistance was confirmed by synergistic assays conducted.

Conclusion: Evidence of carbamate resistance in A. gambiae populations already harbouring resistance to DDT and permethrin is a clear indication that calls for the implementation of insecticide resistance management strategies to combat the multiple resistance identified.
\end{abstract}

Keywords: Carbamate, DDT, Pyrethroids, Insecticide resistance, Urban, Anopheles gambiae mosquitoes, Lagos, Nigeria

\footnotetext{
* Correspondence: dayooduola@yahoo.co.uk

${ }^{1}$ Molecular Entomology and Vector Control Research Laboratory, Public

Health Division, Nigerian Institute of Medical Research, Akoka, Lagos, Nigeria

${ }^{2}$ Department of Zoology, University of Lagos, Akoka, Lagos, Nigeria
} 


\section{Background}

Malaria is a major public health problem and Anopheles gambiae is one of the major vectors of this disease in subSaharan Africa [1]. In most urban communities of Lagos State, A. gambiae mosquitoes are found breeding abundantly in widely available sun-lit stagnant pools of water created by flooding resulting from numerous blocked drains during the rainy season [2]. The abundance of these vector species during the rainy season between April and October usually coincides with the peak of the malaria transmission period. The current best practices in vector control include the use of Long Lasting Insecticide Nets (LLIN), Indoor Residual Spraying (IRS) and Larviciding [3].

In recent times, IRS is being adopted and scaled up to protect the entire household and community members who possibly have no access to treated bed nets. It is also being scaled up to address the problem of slow improvement in the utilization of bed nets in Africa $[4,5]$.

Out of the four classes of insecticides (organochlorine, pyrethroids, carbamates and organophosphates) approved for malaria vector control [6] only the pyrethroid insecticides are recommended for the treatment of bed nets, the others are applied for IRS. In Nigeria, pyrethroids are currently the most widely used insecticides either in the treatment of bed nets or for IRS. Its usage as an insecticide of choice for malaria vector control purposes relies on their known efficacy at low dosage, relative safety for humans and non-target organisms, excito-repellent properties, rapid rate of knock-down and residual killing effects [7]. The lower cost of procurement and application when compared to carbamate is another reason for its choice during vector control programmes [8]. Though the World Health Organisation (WHO) has approved the use of dichlorodiphenyltrichloroethane (DDT) for public health [9], the non usage of DDT by some countries may not be farfetched from issues on human and environmental safety [10]. Insecticide mosaic and rotational use of different classes has been proposed for resistance management [11]. With the widespread resistance to pyrethroid and organochlorine, carbamate is one of the possible alternatives that can be considered to combat pyrethroid-DDT resistance, mainly because of its separate mode of action. Pyrethroids and DDT insecticides share the same target site; hence resistance to one class may possibly indicate resistance to another. In terms of cost of application on a house to house basis, DDT still remains the least expensive of all the three classes of insecticides [8].

Unfortunately, resistance of Anopheles species to several classes of insecticide; DDT, carbamates, organophosphates and pyrethroids has been reported in several African countries [12]. In Nigeria, resistance of Anopheles gambiae, the major malaria vector to organochlorine and pyrethroid insecticides is well documented [13-15]. However, there is paucity of information on the insecticide resistance status of the field strain of A. gambiae in Nigeria to carbamate insecticide.

The objective of this study is to provide information on the susceptibility status of Anopheles gambiae to carbamate that has not been used in vector control and also to investigate the possibility of co-existence of multiple insecticide resistance in the same population of $\mathrm{A}$. gambiae that was previously reported to be resistant to permethrin and DDT [15]. It is hoped that findings from this study will promote and improve effective vector control decision making.

\section{Methods}

\section{Study area}

The study was carried out in 11 communities from 10 Local Government Areas (LGAs) in Lagos State ( $3^{\circ} 24^{\prime} \mathrm{E}$ and latitude $6^{\circ} 27^{\prime} \mathrm{N}$ ). Lagos is a city in south-western Nigeria (Figure 1). The study communities and corresponding LGAs are shown in Table 1. The Lagos metropolitan area spreads over much of Lagos State $\left(3500 \mathrm{~km}^{2}\right)$, and occupies Lagos Island, Ikoyi Island, and Victoria Island, as well as a large area on the mainland, all connected by a series of bridges and freeways. Lagos is Nigeria's largest city, chief port, and principal industrial, economic and cultural center. Lagos has a very diverse and fast-growing population, resulting from heavy and ongoing migration to the city from all parts of Nigeria as well as neighbouring countries for trading and employment opportunities.

\section{Mosquito larval collection and rearing}

Anopheles gambiae s.l. larvae were collected in 11 study communities namely: Badagry, Mile 2, Isaga, Bariga, Ikorodu, Ikoyi, Mile 12, Egbeda, Iyana Iba, Okokomaiko, Isheri-Ikotun selected randomly, from June to August 2010. At each locality chosen, Anopheline larvae were collected from various natural breeding sites including ground pools, gutters, puddles and abandoned potholes. Water was scooped using a plastic scoop and poured into small transparent plastic bowls. A strainer was used to sieve and pool together the third and fourth instar larvae in order to have sufficient adult emergence of the same physiological age. The bowls were scrutinized for presence of unwanted organisms or predators; if any were found, a pipette was used to remove them. The Global Positioning System (GPS) was used to establish the coordinates of the study sites. The mosquito larvae collected were transported in well labelled plastic bottles to The Molecular Entomology and Vector Control Unit Insectary at the Nigerian Institute of Medical Research, Yaba Lagos where they were maintained at $28 \pm 2 \mathrm{C}$ and $72 \pm 5 \%$ relative humidity. 


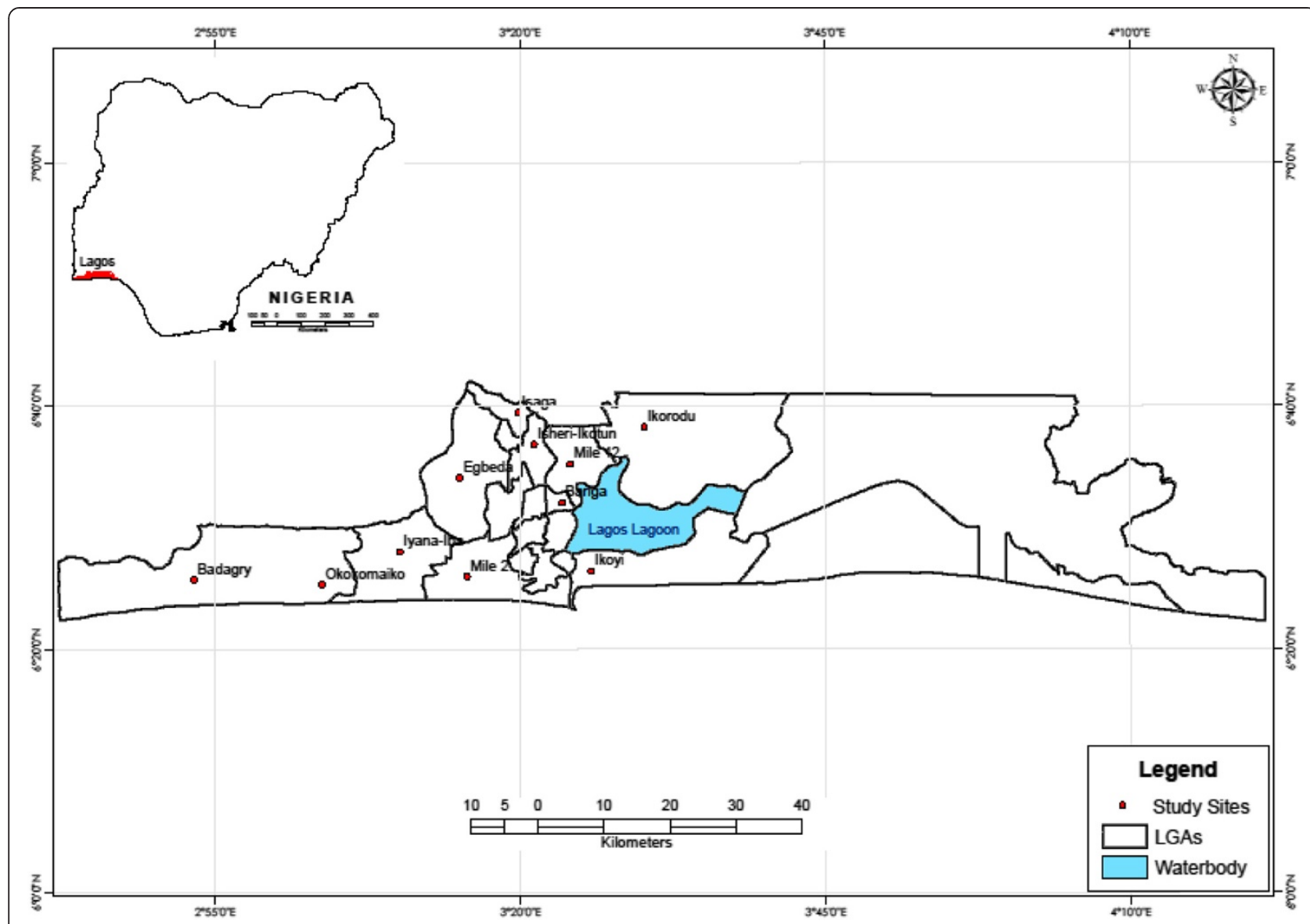

Figure 1 Map of Lagos showing the study localities, displayed in the inset is the map of Nigeria.

\section{Mosquito identification}

The mosquitoes exposed in the tests were examined under the dissecting microscope (Olympus SZ 40) and identified to species level using morphological identification keys [16].

\section{Insecticide susceptibility tests}

Insecticide susceptibility tests were performed using the WHO standard procedures and test kits for adult mosquitoes [17]. Test papers impregnated with recommended diagnostic concentrations of $4 \%$ DDT, $0.05 \%$ deltamethrin, $0.1 \%$ propoxur and $0.1 \%$ bendiocarb were used. The Kisumu reference strain susceptible to all insecticide classes was used to establish the potency of the treated papers before the exposures were carried out using wild samples. Tests were carried out using 2-3 day old, non-blood fed female mosquitoes. For each insecticide, 3-4 replicates of 20 to 25 females were exposed to the insecticide-impregnated test papers in the test tubes for 1 hour. Considering the homogeneous identity of populations of A. gambiae observed in Lagos, additional tests were carried out to determine the effect of $\mathrm{PBO}$ synergist combinations with bendiocarb and propoxur on the mortality of A. gambiae collected. Adult female mosquitoes were pre-exposed to $4 \%$ PBO paper for 1hour following which they were immediately exposed to bendiocarb and propoxur for another 1hour. Final mortality was recorded after 24 hours and the results compared with those without $\mathrm{PBO}$. Sub-populations were also exposed to DDT and deltamethrin to confirm the susceptibility status of the population to both insecticides as earlier reported. The number of mosquitoes that were knockdown was recorded every ten minutes during the 1hour exposure period. Mosquitoes were then transferred into holding tubes and supplied with a $10 \%$ sugar solution. Mortality was recorded 24 hours after exposure. Controls were also set up by exposing the field strain to untreated papers. The tests results were discarded if mortality in the control group was over 20\%. If it was between 5 and 20\%, mortality rates were corrected using Abbott's formula [18]. Resistant and susceptible mosquitoes were preserved separately in eppendorf tubes filled with desiccated silica gel.

DNA extraction, molecular identification, $k d r-w / e$ and ace$1^{\mathrm{R}}$ mutations of acetyl cholinesterase gene

Genomic DNA was extracted from sub samples of mosquitoes that were randomly selected from a pool of 
Table 1 Knockdown times (KDTs) for Anopheles gambiae s.l.in urban Lagos after exposure to different insecticides

\begin{tabular}{|c|c|c|c|c|c|c|c|c|c|c|}
\hline \multirow[b]{2}{*}{ LGA } & \multirow[b]{2}{*}{ Study sites } & \multicolumn{3}{|c|}{ DDT $(4 \%)$} & \multicolumn{3}{|c|}{ Deltamethrin(0.05\%) } & \multicolumn{3}{|c|}{ Propoxur $(0.1 \%)$} \\
\hline & & $\mathbf{n}$ & $\begin{array}{l}\mathrm{KDT}_{50} \\
(95 \% \mathrm{Cl})\end{array}$ & $\begin{array}{c}\mathrm{KDT}_{95} \\
(95 \% \mathrm{Cl})\end{array}$ & $\mathbf{n}$ & $\begin{array}{l}\mathrm{KDT}_{50} \\
(95 \% \mathrm{Cl})\end{array}$ & $\begin{array}{c}\mathrm{KDT}_{95} \\
(95 \% \mathrm{Cl})\end{array}$ & $\mathbf{n}$ & $\begin{array}{l}\mathrm{KDT}_{50} \\
(95 \% \mathrm{Cl})\end{array}$ & $\begin{array}{c}\mathrm{KDT}_{95} \\
(95 \% \mathrm{Cl})\end{array}$ \\
\hline Badagry & Badagry & 82 & a & a & 80 & $\begin{array}{c}31.31 \\
(22.65-45.58)\end{array}$ & $\begin{array}{c}96.21 \\
(59.56-411.52)\end{array}$ & 60 & a & a \\
\hline Amuwo Odofin & Mile 2 & 60 & a & a & 60 & $\begin{array}{c}46.03 \\
(39.23-57.71)\end{array}$ & $\begin{array}{c}124.36 \\
(87.60-254.63)\end{array}$ & 60 & a & a \\
\hline Ifako ljaiye & Isaga & 63 & a & a & 60 & $\begin{array}{c}53.20 \\
(47.21-62.46)\end{array}$ & $\begin{array}{c}171.11 \\
(126.43-275.01)\end{array}$ & 60 & a & a \\
\hline Somolu & Bariga & & $b$ & $b$ & 88 & $\begin{array}{c}39.49 \\
(36.30-42.91)\end{array}$ & $\begin{array}{c}59.82 \\
(54.65-67.73)\end{array}$ & 87 & $\begin{array}{c}59.90 \\
(56.32-64.76)\end{array}$ & $\begin{array}{c}91.88 \\
(83.41-104.00)\end{array}$ \\
\hline Ikorodu & Ikorodu & 80 & $\begin{array}{c}12 . \\
(11.25-14.88)\end{array}$ & $\begin{array}{c}19.78 \\
(48.96-78.73)\end{array}$ & 93 & $\begin{array}{c}49.54 \\
(47.47-51.87)\end{array}$ & $\begin{array}{c}73.84 \\
(69.48-79.60)\end{array}$ & 83 & $\begin{array}{c}42.52 \\
(40.72-44.38)\end{array}$ & $\begin{array}{c}62.21 \\
(59.08-66.18)\end{array}$ \\
\hline Eti Osa & Ikoyi & 80 & $b$ & $b$ & 85 & $\begin{array}{c}48.13 \\
(45.64-51.10)\end{array}$ & $\begin{array}{c}88.32 \\
(78.21-105.15)\end{array}$ & 84 & $\begin{array}{c}42.97 \\
(40.03-46.01)\end{array}$ & $\begin{array}{c}60.80 \\
(56.25-67.78)\end{array}$ \\
\hline Kosofe & Mile 12 & 80 & $b$ & $b$ & 100 & $\begin{array}{c}50.99 \\
(39.13-42.92)\end{array}$ & $\begin{array}{c}67.96 \\
(62.62-75.87)\end{array}$ & 80 & $\begin{array}{c}38.93 \\
(37.17-40.75)\end{array}$ & $\begin{array}{c}58.84 \\
(55.82-62.63)\end{array}$ \\
\hline Alimoso & Egbeda & 111 & a & a & 90 & $38.40(35.99-41.10)$ & $\begin{array}{c}91.69 \\
(80.17-109.19)\end{array}$ & 88 & a & a \\
\hline Ojoo & Iyana-Iba & 86 & a & a & 97 & $\begin{array}{c}50.47 \\
(47.44-54.30)\end{array}$ & $\begin{array}{c}106.63 \\
(91.69-132.05)\end{array}$ & 89 & $\begin{array}{c}38.40 \\
(36.35-40.58)\end{array}$ & $\begin{array}{c}75.66 \\
(68.32-86.40)\end{array}$ \\
\hline Ojoo & Okokomaiko & 80 & c & c & 87 & $\begin{array}{c}41.32 \\
(38.60-44.51)\end{array}$ & $\begin{array}{c}101.62 \\
(87.25-124.64)\end{array}$ & 94 & $\begin{array}{c}46.03 \\
(43.53-48.96)\end{array}$ & $\begin{array}{c}92.55 \\
(81.57-110.02)\end{array}$ \\
\hline Alimoso & Isheri-Ikotun & 80 & c & c & 93 & a & $\begin{array}{c}244.45 \\
(170.49-438.37)\end{array}$ & 88 & $\begin{array}{c}55.18 \\
(46.38-97.48)\end{array}$ & $\begin{array}{c}89.97 \\
(66.69-83.99)\end{array}$ \\
\hline Total & & 802 & & & 933 & & & 873 & & \\
\hline
\end{tabular}

$\mathrm{n}=$ numbers of mosquitoes exposed $\mathrm{a}=50 \%$ knockdown was not obtained within the 60 minutes of exposure period $\mathrm{b}=$ No knockdown effect. $\mathrm{c}=$ Lower and upper confidence limits could not be estimated due to large $\mathrm{KdT}$ value.

susceptible and resistant populations from each study location [19]. Extracted DNA was subjected to Polymerase Chain Reaction - Restriction Fragment Length Polymorphism (PCR- RFLP) assay to simultaneously identify members of Anopheles gambiae complex and the molecular forms [20]. The $k d r-w / e$ and insensitive G119S (Ace. $1^{R}$ ) point mutation recognition assays were carried out on at least 20 sub - samples selected from each population [21-23].

\section{Interpretation of data and statistical analysis}

Percentage knockdown to the three insecticides and percentage mortality were determined for the mosquitoes from each of the study localities. The WHO [17] criteria was used to evaluate the resistance/susceptibility status of the mosquitoes. By the criteria, resistance is indicated by mortality rates of less than $80 \%$ at $24 \mathrm{hrs}$ post exposure, while mortality rates greater than $97 \%$ are indicative of susceptibility. Mortality rates between $80-97 \%$ indicate that resistance is suspected [17]. For probit analysis, knockdown times for $50 \%$ and $95 \%$ of the test population were estimated by the log time probit model using the Microsoft Excel Software 2007. The $k d r$ allelic frequencies were calculated using the Hardy -Weinberg equation. Unpaired sample $t$-test was also used to determine the significant difference in mortality rates between each of the insecticides used using the SPSS software (version 15.0)

\section{Results}

\section{Insecticide susceptibility}

Overall, 2900 adult female mosquitoes were reared to adults from larval collections made in eleven localities. Of these, 2608 were morphologically identified as $A$. gambiae s.l and exposed to discriminating doses of DDT, deltamethrin and propoxur (Table 1). All the field populations of $A$. gambiae s.l were resistant to DDT with mortality rates $(\leq 16 \%)$ after a 24 hour recovery period (Figure 2). Resistance to deltamethrin was observed in A. gambiae populations in five localities; Isaga, Ikorodu, Ikoyi, Iyana Iba and Isheri - Ikotun. Mortality rates ranging between $25-77 \%$ from propoxur exposure indicate resistance in ten localities excluding Isaga where resistance was suspected. Resistance to deltamethrin was also suspected in populations from Badagry, Mile 2, Bariga, Egbeda, Mile 12 and Okokomaiko, where mortality rates ranged between $80-95 \%$. In summary, multiple resistance to three different classes of insecticides was observed in populations from five localities (Bariga, Ikorodu, Ikoyi, Iyana Iba and Isheri -Ikotun) (Figure 2). 


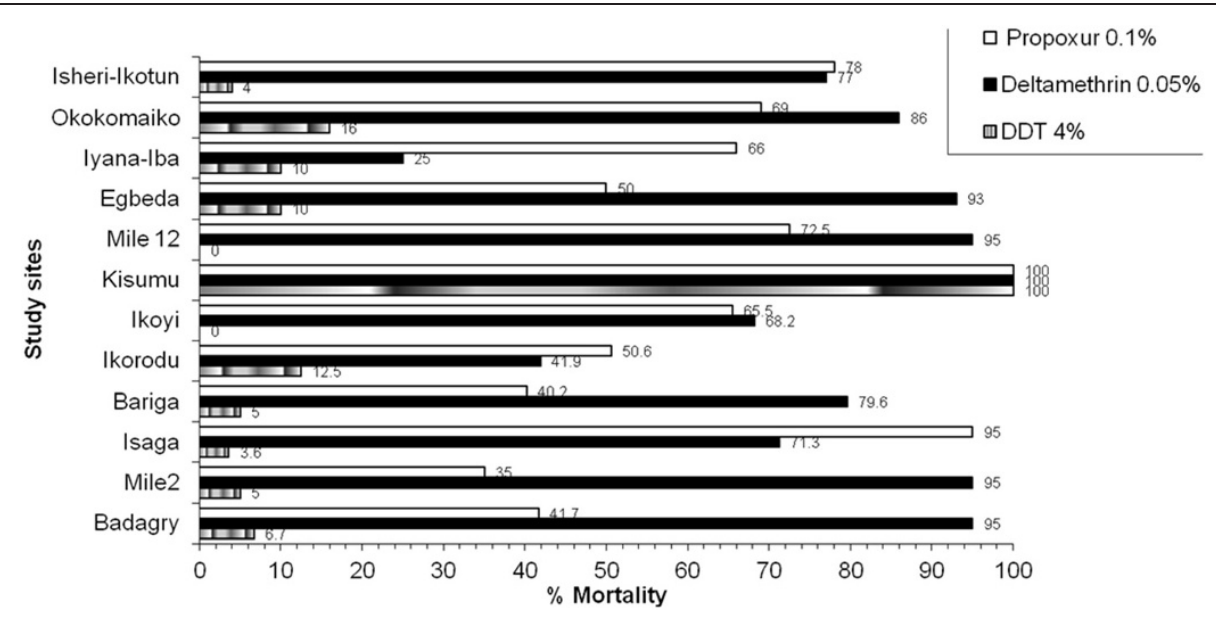

Figure 2 Insecticide susceptibility status of Anopheles gambiae s.l in urban Lagos compared with the Kisumu strain.

Two populations of $A$. gambiae from Ikoyi and Mile 12 showed no mortality after one hour exposure to DDT. The overall results based on the WHO criteria for evaluating resistance suggests that the resistance status of Anopheles gambiae in urban Lagos vary with none of the populations exhibiting full susceptibility to any of the 3 insecticides tested.

\section{Knockdown effect of the insecticides}

Percentage knockdown at 10 minute intervals was significantly higher in the susceptible Kisumu strain for all insecticides tested compared to all other samples from different localities $(\mathrm{P}<0.05)$ in all cases. The logtime probit model used to estimate the KDT ${ }_{50} \&$ KDT 95 values (percentage knockdown with time) for DDT in Badagry, Mile 2, Isaga, Egbeda, Iyana Iba, Okokomaiko and Isheri-Ikotun study localities (Table 1) indicated values that were unrealistic being greater than the mandatory 60 minutes recommended by the World Health Organization. All other KDT 50 and KDT 95 values of $A$. gambiae populations exposed to deltamethrin and propoxur within an hour were higher than those for the Kisumu susceptible by a factor of about 34 fold. Knock down time values higher than these are indicative of a very high level of resistance.

\section{Mortality against bendiocarb and propoxur in populations pre-exposed to piperonyl butoxide (PBO) synergist}

The mortality rate observed in A. gambiae populations exposed to propoxur was lower ( $\leq 70 \%)$ compared with mortality rates $(\leq 80 \%)$ in populations exposed to bendiocarb. However, a full susceptibility was observed in exposures to both insecticides when the A. gambiae populations were pre-exposed to $\mathrm{PBO}$ synergist (Figure 3).
Species identification, molecular forms and frequencies of $k d r$ and ace $-1^{R}$ mutations

All mosquito samples (susceptible and resistant) drawn at random and analysed by species - specific PCR assays were identified as predominantly A. gambiae s.s and all belonged to the molecular $M$ form (Table 2). The knockdown resistance gene $(k d r)$ was detected in all the Anopheles populations at various allelic frequencies ranging between $45 \%-77 \%$ (Table 3). The genotypic frequencies showed that $134(61 \%)$ and 79 (35.9\%) mosquitoes were homozygous for the susceptible and resistance alleles. Only $4(1.8 \%)$ carried the heterozygous alleles. The $k d r(e)$ and ace- $1^{\mathrm{R}}$ mutation was absent in all the populations.

\section{Discussion}

This study demonstrates the occurrence of resistance to deltamethrin, DDT and propoxur insecticides in populations of A. gambiae in urban Lagos. Previous studies have reported widespread resistance to DDT and pyrethroids in Anopheles gambiae from sub - Saharan Africa $[24,25]$. The existence of DDT resistance in all the urban communities surveyed is a confirmation of the spatiotemporal spread of DDT resistance in the urban communities of Lagos.

The co - occurrence of both DDT and deltamethrin resistance in the same $A$. gambiae population suggest a similar mechanism modulating for both pyrethroid and DDT resistance since both insecticides act on the same target site. This was confirmed with the identification of the $k d r$ resistance mechanisms in all the communities. The discovery of carbamate resistance in 10 out of the 11 communities confirmed mosquito populations harbouring resistance to 3 classes of insecticides. Similar reports from studies carried out using field strains of A. gambiae populations in Guinea Conakry [26] and Congo [27] have 


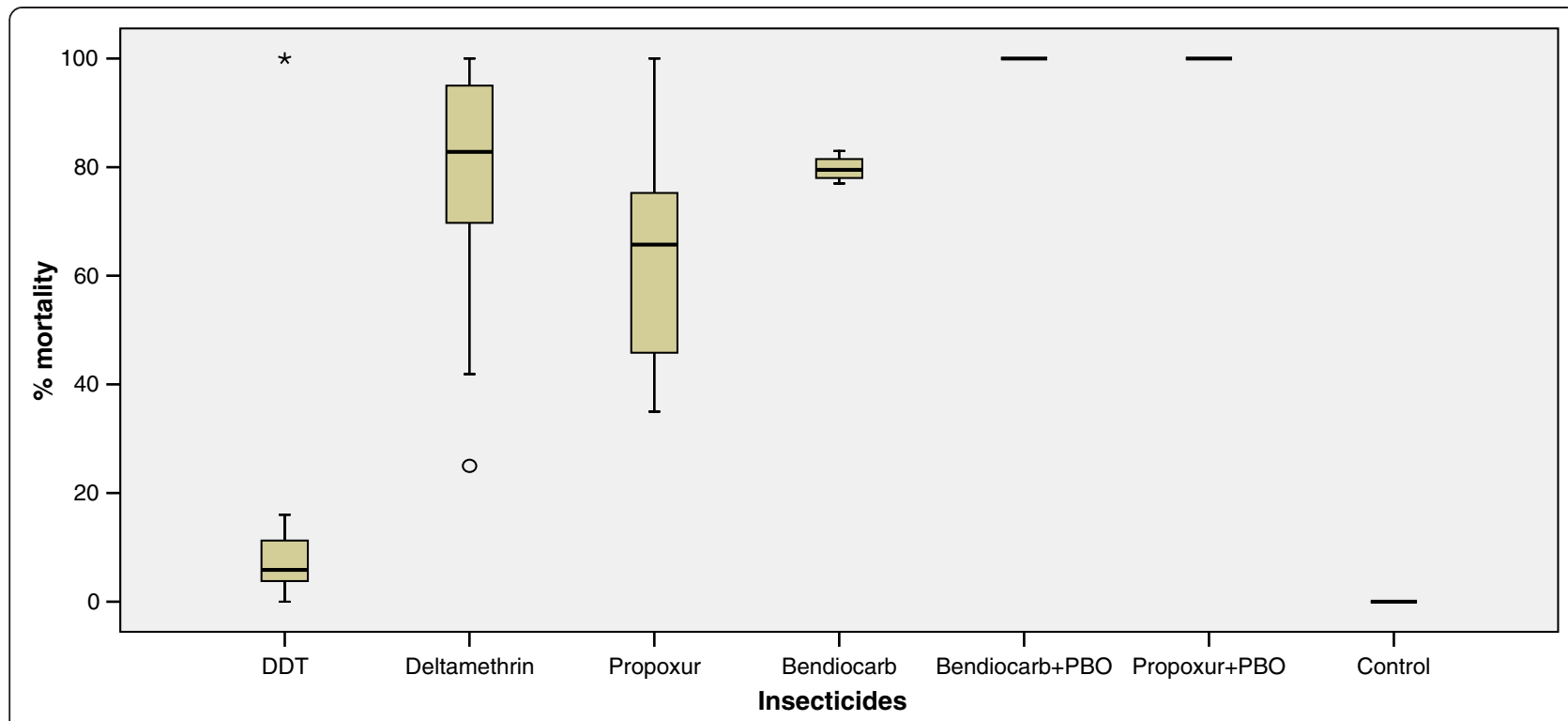

Figure 3 Twenty- four hour post exposure mortalities of synergised and unsynergised A. gambiae exposed to different insecticides.

demonstrated the occurrence of resistance to more than one class of insecticide. However, this is a first report of carbamate resistance in $A$. gambiae s. $s$ in Nigeria, and evidence of its occurrence in populations already harbouring resistance to pyrethroids and organochlorines.

The existence of resistance to three classes of insecticides in populations of $A$. gambiae in urban Lagos is worrisome and an indication of the threatened sustainability of malaria vector control programmes utilising any of these insecticides. The major challenge arising from these results is that the choice of carbamate may

Table 2 Species composition and frequency of molecular forms of $A$. gambiae mosquitoes exposed to different insecticides

\begin{tabular}{|c|c|c|c|c|c|}
\hline & Insecticides & $\mathbf{N}$ & $\begin{array}{c}\text { A.gambiae } \\
\text { S.S }\end{array}$ & $\begin{array}{c}M \\
\text { forms }\end{array}$ & $\begin{array}{c}S \\
\text { forms }\end{array}$ \\
\hline & DDT & 110 & 97 & 96 & 0 \\
\hline \multirow{4}{*}{$\begin{array}{l}\text { Resistant } \\
\text { samples }\end{array}$} & Deltamethrin & 95 & 91 & 91 & 0 \\
\hline & Propoxur & 103 & 98 & 94 & 0 \\
\hline & & $\begin{array}{c}308 \\
(54.1 \%)\end{array}$ & & & \\
\hline & DDT & 54 & 52 & 49 & 0 \\
\hline \multirow{4}{*}{$\begin{array}{l}\text { Susceptible } \\
\text { samples }\end{array}$} & Deltamethrin & 98 & 93 & 87 & 0 \\
\hline & Propoxur & 110 & 97 & 93 & 0 \\
\hline & & $\begin{array}{c}262 \\
(45.9 \%)\end{array}$ & & & \\
\hline & & 570 & $528(92.6 \%)^{*}$ & 510 & 0 \\
\hline
\end{tabular}

*Eight percent of the samples failed to amplify.

$N=$ Number of samples analysed. not be a suitable alternative in the event of switching to another class of insecticide.

The identification of $A$. gambiae s.s and the molecular M forms in all samples analysed in Lagos is a clear indication of the predominance of these species in the urban communities where this study was carried out. These findings agree with earlier studies, which reported predominantly pure collections of $A$. gambiae s.s in south-western, Nigeria $[13,28]$. Interestingly, the species composition, the prevailing molecular $\mathrm{M}$ forms and the widespread incidence of the $k d r$ resistance mechanism in the $A$. gambiae

Table 3 The allelic frequencies of $k d r$ mutation in molecular M forms of A.gambiae s.s in the study localities

\begin{tabular}{lccccc}
\hline \multicolumn{1}{c}{$\begin{array}{c}\text { Study } \\
\text { localities }\end{array}$} & ${ }^{*} \mathbf{N}$ & \multicolumn{2}{c}{$\boldsymbol{k d r}$ mutation genotypes } & $\begin{array}{c}\text { Allelic } \\
\text { frequency } \\
\text { SS }\end{array}$ \\
\hline Badagry & 20 & 14 & 0 & 6 & 0.55 \\
Mile 2 & 20 & 14 & 0 & 6 & 0.55 \\
Isaga & 20 & 8 & 0 & 12 & 0.77 \\
Bariga & 20 & 10 & 0 & 8 & 0.63 \\
Ikorodu & 20 & 9 & 0 & 11 & 0.74 \\
Ikoyi & 20 & 11 & 0 & 8 & 0.63 \\
Mile 12 & 20 & 14 & 0 & 6 & 0.55 \\
Egbeda & 20 & 13 & 0 & 7 & 0.59 \\
lyana-Iba & 20 & 14 & 2 & 4 & 0.45 \\
Okokomaiko & 20 & 14 & 0 & 6 & 0.55 \\
Isheri-Ikotun & 20 & 14 & 2 & 4 & 0.45 \\
Total & $\mathbf{2 2 0}$ & $\mathbf{1 3 4}(\mathbf{6 0 . 9 \% )}$ & $\mathbf{4 ( 1 . 8 \% )}$ & $\mathbf{7 9 ( 3 5 . 9 \% )}$ & \\
\hline
\end{tabular}

${ }^{*} \mathrm{~N}=$ Number of $A$. gambiae s.s ' $\mathrm{M}$ ' form analysed. 
populations in Lagos share a strong similarity with A. gambiae populations from Ladji; an urban community in Benin Republic [29]. It is possible that there is a strong dispersal of mosquito species between Lagos and Benin republic owing to their location on the same transport corridor.

The higher disparity in mortalities observed in deltamethrin exposure compared with DDT suggest that the $k d r$ alone may not be responsible for the resistance observed in these populations. Hence, the involvement of an additional operative resistance mechanism is possible. The leucine-serine point mutation $(k d r-e)$, high levels of glutathione s-transferase and oxidase activities have been reported to confer higher resistance to DDT over pyrethroids [22,30]. In this study, the kdr-e mutation was found to be absent in the A. gambiae populations analysed. However, the synergistic assay confirmed the activity of metabolic enzymes, though not characterised, but may have increased resistance to DDT. Equally, the operative resistance mechanism responsible for the insensitivity of wild A. gambiae s.s to propoxur and bendiocarb was not as a result of mutation in the acetylcholinesterase target site reported from studies conducted in Burkina Faso and Cote d'Ivoire [31,32]. This was confirmed with the full restoration of susceptibility to carbamate in A. gambiae subpopulations pre-exposed to PBO synergists. Similar occurrence of carbamate resistance not associated with the G119S mutation has been reported in A. gambiae s.s from Congo [27], Anopheles arabiensis from Ethiopia [33] and in A. funestus from Mozambique [34].

Factors such as agricultural use of carbamate cannot be linked to selection for carbamate resistance as previously mentioned by other authors [29,32]. This is solely because the localities where these studies were conducted were non-agricultural areas. However, there is convincing evidence of the use of organophosphate based "locally made insecticides" by householders for personal protection in many of these localities (unрublished results). Hence, the carbamate resistance recorded may possibly have resulted from the elevated levels of esterases, which have been reported to be a primary mechanism involved in organophosphate and carbamate resistance $[35,36]$.

\section{Conclusions}

Carbamate may not be an alternative insecticide to substitute for the pyrethroid/DDT resistance occasioned in A. gambiae populations in Lagos. Hence, there is a need to incorporate resistance and integrated vector management approaches to malaria vector control in urban communities of Lagos, Nigeria.

\section{Competing interests}

The authors declare that they have no competing interests.

\section{Acknowledgements}

We would like to thank the following; Emmanuel Otegbayo, Olive Okafor, Olufunmi Jimoh for providing technical assistance in the field and laboratory towards the completion of this study. This investigation received partial financial support under the MIM project A60039 awarded to TSA through the UNICEF/UNDP/World Bank/WHO special programme for Research and Training in Tropical Diseases.

\section{Authors' contributions}

OAO and TSA conceived and designed the study, AOO and ETI implemented the field study and carried out the susceptibility tests, MKO and AAA carried out the laboratory studies. MKO and JBO collated and analysed the data. AOO drafted the manuscript while TSA and OAO helped in editing the overall manuscript. All authors read and approved the final version of the manuscript.

Received: 12 March 2012 Accepted: 11 June 2012

Published: 11 June 2012

\section{References}

1. Gillies MT, Coetzee M: A supplement to the Anophelinae Africa South of the Sahara (Afrotropical region), Johannesburg, South Africa. S Afr Inst Med Res 1987, 55:1-143.

2. Awolola TS, Oduola AO, Obansa JB, Chukwura NJ, Unyimadu JP: Anopheles gambiae s.s breeding in polluted water bodies in urban Lagos, southwestern Nigeria. J Vector Borne Dis 2007, 44:241-244.

3. Beier JC, Keating J, Githure JI, Macdonald MB, Impoinvil DE, Novak RJ: Integrated vector management for malaria control. Malar J 2008, 7(supp 1). doi:articleS4 10.1186/1475-2875-7-S1- S4.

4. Atieli HE, Zhou G, Afrane Y, Lee M, Mwanzo I, Githeko AK, Yan G: Insecticide-treated net (ITN) ownership, usage, and malaria transmission in the highlands of western Kenya. Parasit Vectors 2011, 4:113.

5. ScienceDaily. Retrieved July 19, 2011. http://www.sciencedaily.com/releases/ 2008/11/081118094632.htm.

6. Devine GJ, Ogusuku E: Adaptability is key when monitoring insecticide resistance. Bull World Health Organ 2009, 87:887-887.

7. Zaim M, Aitio A, Nakashima N: Safety of pyrethroid-treated mosquito nets. Med Vet Entomology 2000, 14:1-5.

8. Walker K: Cost comparison of DDT and alternative insecticides for malaria control. Med Vet Entomology 2000, 14:345-354.

9. WHO: Indoor Residual Spraying: Use of Indoor Residual Spraying for Scaling Up Global Malaria Control and Elimination: WHO Position Statement. Geneva: Global Malaria Programme, World Health Organization; 2006.

10. Eskenazi B, Chevrier J, Rosas LG, Anderson HA, Bornman MS, Bouwman H, Chen A, Cohn BA, de Jager C, Henshel DS, Leipzig F, Leipzig JS, Lorenz EC, Snedeker SM, Stapleton D: The Pine River statement: human health consequences of DDT use. Env Health Persp 2009, 117(9):1359-1367.

11. Brogdon WG, MC Allister JC: Insecticide Resistance and Vector Control. Emerging Infect Dis 1998, 4(4):606-613.

12. Kristan M, Fleischman H, Della Torre A, Stich A, Curtis CF: Pyrethroid resistance/Susceptibility and differential urban/rural distribution of Anopheles arabiensis and A. gambiae s.s malaria vectors in Nigeria and Ghana. Med Vet Entomol 2003, 17:326-332.

13. Awolola TS, Brooke BD, Hunt RH, Coetzee M: Resistance of the malaria vector Anopheles gambiae s.s to pyrethroid insecticide in south westernNigeria. Ann Trop Med and Parasitology 2002, 96:849-852

14. Awolola T, Oduola A, Oyewole IO, Obansa JB, Amajoh C, Koekemoer L, Coetzee M: Dynamics of knockdown pyrethroid insecticide resistance alleles in a field population of Anopheles gambiae s.s. in southwestern Nigeria. J Vect Borne Dis 2007, 44:181-188.

15. Oduola AO, Obansa JB Ashiegbu CO, Adeogun A, Otubanjo OA, Awolola TS: High level of DDT resistance in the malaria mosquito: Anopheles gambiae s.l. from rural, semi urban and urban communities in Nigeria. $J$ Rural Trop Pub Health 2010, 9:114-120.

16. Gillies MT, Coetzee M: A Supplement to the Anophelinae of Africa South of the Sahara. In Publication of South African Institute of Med Res 1987, 55:1-143.

17. World Health Organisation: Test procedures for Insecticide Resistance in Malaria Vectors, Bio-efficacy and Persistence of Insecticides on Treated Surfaces. (WHO/CDS/CPC/MAL/98.12). Switzerland: WHO Geneva; 1998. 
18. Abott WS: A method of computing the effectiveness of an insecticide. J Am Mosa Control Assoc 1987, 3:302-303.

19. Collins FH, Mendez MA, Rasmussen MO, Mehaffey PC, Besansky NJ, et al: A ribosomal RNA gene probe differentiates member species of the Anopheles gambiae complex. AmJTrop Med Hyg 1987, 37:37-41.

20. Fanello C, Della Torre A, Santolamazza F: Simultaneous identification of species and molecular forms of the Anopheles gambiae complex by PCR-RFLP. Med Vet Entomol 2002, 16:461-464.

21. Martinez-Torres D, Chandre F, Williamson MS, Darriet F, Berge JB, Devonshire AL, Guillet P, Pasteur N, Pauron D: Molecular characterization of pyrethroid knockdown resistance $(k d r)$ in the major malaria vector Anopheles gambiae s.s. Insect Mol Biol 1998, 7:179-184.

22. Ranson $\mathrm{H}$, Jensen $B$, Vulule JM, Wang $X$, Hemingway J, Collins FH: Identification of a point mutation in the voltage-gated sodium channel gene of Kenyan Anopheles gambiae s.s. associated with resistance to DDT and pyrethroids. Ins Mol Biol 2000, 9:491-497.

23. Weill M, Malcolm C, Chandre F, Mogensen K, Berthomieu A, Marquine M, Raymond M: The unique mutation in Ace-1 giving high insecticide resistance is easily detectable in mosquito vectors. Insect Mol Biol 2004, 13:1-7.

24. Himeidan YE, Abdel Hamid MM, Jones CM, Ranson H: Extensive permethrin and DDT resistance in Anopheles arabiensis from eastern and central Sudan. Parasit Vectors 2011, 4:154

25. Rogan WJ, Chen A: Health risks and benefits of bis(4-chlorophenyl)1,1,1-trichloroethane (DDT). Lancet 2005, 366(9487):763-773.

26. Vezenegho SB, Brooke BD, Hunt RH, Coetzee M, Koekemoer LL: Malaria vector mosquito composition and insecticide susceptibility status in Guinea Conakry, West Africa. Med Vet Entomol 2009, 23:326-334.

27. Koekemoer LL, Spillings BL, Christian RN, Lo TM, Kaiser ML, Norton RAl, Oliver SV, Choi KS, Brooke BD, Hunt RH, Coetzee M: Multiple Insecticide Resistance in Anopheles gambiae (Diptera: Culicidae) from Pointe Noire, Republic of the Congo. Vect. Borne Zoo Dis 2011. doi:10.1089/vbz.2010.0192.

28. Onyabe DY, Vajime CG, Nock IH, Ndams IS, Akpa AU, Alaribe AA, Conn JE: The distribution of $\mathrm{M}$ and $\mathrm{S}$ molecular forms of Anopheles gambiae in Nigeria. Trans R Soc Trop Med Hyg 2003, 97:605-608.

29. Yadouleton AW, Padonou G, Asidi A, Moiroux N, Bio-Banganna S, Corbel Corbel Gbenou D, Yacoubou I, Gazard K, Akogbeto MC: Insecticide resistance status in Anopheles gambiae in southern Benin. Malaria J 2010, 9:83.

30. Ranson H, Jensen B, Wang X, Prapanthadara L, Hemingway J, Collins FH: Genetic mapping of two loci affecting DDT resistance in the malaria vector Anopheles gambiae. Ins Mol Biol 2000, 9:499-507.

31. Ahoua Alou LP, Koffi AA, Adja MA, Tia E, Kouassi PK, Koné M, Chandre F: Distribution of ace-1R and resistance to carbamates and organophosphates in Anopheles gambiae s.s populations from Côte d'Ivoire. Malaria J 2010, 9:167.

32. Dabire KR, Diabate A, Djougbenou A, Ouari A, N'Guessan R, Ouédraogo J, Hougard J, Chandre F, Baldet T: Dynamics of multiple insecticide resistance in the malaria vector Anopheles gambiae in a rice growing area in South- Western Burkina Faso. Malaria J 2008, 7:188.

33. Yewhalaw D, Wassie F, Steurbaut W, Spanoghe P, Van Bortel W, et al: Multiple Insecticide Resistance: An Impediment to Insecticide-based Malaria Vector Control Programme. PLoS One 2011, 6(1):e16066.

34. Cuamba N, Morgan JC, Irving H, Steven A, Wondji CS: High Level of Pyrethroid Resistance in an Anopheles funestus Population of the Chokwe District in Mozambique. PLoS One 2011, 5(6):e11010.

35. Hemingway J, Karunaratne SHPP: Mosquito carboxylesterases: a review of the molecular biology and biochemistry of a major insecticide resistance mechanism. Med. Vet. Entomol 1998, 12:1-12.

36. Hemingway J, Hawkes NJ, McCarroll L, Ranson H: The molecular basis of insecticide resistance in mosquitoes. Insect Biochem Mol Biol 2004, 34 (7):653-665

doi:10.1186/1756-3305-5-116

Cite this article as: Oduola et al:: Evidence of carbamate resistance in urban populations of Anopheles gambiae s.s. mosquitoes resistant to DDT and deltamethrin insecticides in Lagos, South-Western Nigeria. Parasites \& Vectors 2012 5:116.

\section{Submit your next manuscript to BioMed Central and take full advantage of:}

- Convenient online submission

- Thorough peer review

- No space constraints or color figure charges

- Immediate publication on acceptance

- Inclusion in PubMed, CAS, Scopus and Google Scholar

- Research which is freely available for redistribution 